\title{
Heavy Loaded Parts of Petrochemical Equipment Destruction Cause Investigation
}

\author{
A B LAPTEV ${ }^{1}$, S A NAPRIENKO ${ }^{1}, \mathrm{R}_{\mathrm{ZH}}$ AKHIYAROV ${ }^{2}, \mathrm{~A} \mathrm{~V} \mathrm{GOLUBEV}^{3}$ \\ ${ }^{1}$ FSUE "All-Russian Research Institute of Aviation Materials", \\ 17, Radio str., Moscow, 105005, \\ RUSSIA \\ ${ }^{2} \mathrm{OOO}$ "Tehekspertiza", \\ 12, office 1, Dauta Yultya st., Ufa, 450081, \\ RUSSIA \\ ${ }^{3}$ FSBEI of Higher Education "Ufa State Oil Technical University", \\ 1, Kosmonavtov st., Ufa, 450064, \\ RUSSIA
}

\begin{abstract}
The problem of using specialized passivating metals and alloys lies in a rather narrow range of the protecting film performance. With a slight change in operating conditions, the film is destroyed and an avalanche-like process of local corrosion begins at the place of film breakdown. A sequence has been developed for determining parts destruction causes, including a sequential analysis of operating conditions; nature of the part destruction; corrosion products composition; phase inversion in the alloy during overheating or mechanical stress in the part.
\end{abstract}

Key-Words: petrochemical equipment, metal, alloy during overheating, mechanical stress

Received: March 27, 2021. Revised: November 4, 2021. Accepted: December 12, 2021.Published: January 5, 2022.

\section{Introduction}

Intensification of oil production and processing, toughening of oil and hydrocarbon production and processing regimes lead to widespread use of materials with higher strength and anticorrosive properties. The process of creating new materials is moving towards the production of high alloys based on iron, nickel, titanium and aluminum. An increase in strength characteristics and, at the same time, high resistance to corrosion destruction leads to the creation of passivating multiphase alloys [1]. In such alloys, the forming passive surface film creates a sufficient barrier for corrosion dissolution of the metal. The hardening phases formed in the course of smelting or heat treatment significantly increase the strength of the alloy [2]. Particularly critical parts and structures are made of this type of materials: compressor pump impellers, measuring equipment in the fields, turbine shafts and blades in hydrocarbons transport, furnace coils in petrochemical industries. Breakdowns of such critical units and parts in the petrochemical complex lead not only to significant economic damage, but also to disasters with human casualties and severe environmental consequences. [3].

Modern methods of calculating mechanism's parts function are performed without taking into the account the influence of external environment and the duration of its impact. An isotropic medium with a penny-shaped crack is considered in [4]. Research of the dependencies of the crack radius, opening and pressure distributions on the crack surface on time, fluid viscosity, and fracture toughness of the medium is performed in [5]. Investigation of crack formation and fracture processes with porosity and various types of loads taken into consideration $[6,7]$ also do not allow to evaluate the long-term operation of mechanism parts under environmental influence.

The problem of using specialized passivating metals and alloys lies in a rather narrow range of the protective film performance. With a slight change in the flow rate, $\mathrm{pH}$ and composition of the medium, the ingress of an abrasive, a decrease in the density of hydrocarbons and cavitation, the use of chemicals or other factors, the film is destroyed and an avalanche-like process of local corrosion begins at the place of breakdown of the film, the formation of pits, delamination, cracks and, as a result, destruction of the part.

A particularly important factor in parts performance ensuring is the compliance of the technology and composition in the manufacture of the alloy, the quality of the parts and, correspondingly, the incoming inspection of materials and the final inspection of finished parts. 


\section{Research Results}

As an example of assessing the discrepancy between alloy composition norms and the lack of quality control of manufacturing, one can consider destruction causes analysis of the heat exchanger tube [8].

In this work, we investigated the material of the tube of the shell-and-tube heat exchanger. Within 90 days, after the installation and the start of operation of the heat exchanger, numerous through-corrosive damage appeared on the newly mounted 20 steel tubes.

The main reason for the low corrosion resistance of the pipe material has been established - these are the so-called HCNI (easily hydrolyzed inclusions of alkali metal compounds). The calculated average density of such inclusions is $13 \mathrm{pcs} / \mathrm{mm}^{2}$. This is almost an order of magnitude higher than the regulated value $\left(2 \mathrm{pcs} / \mathrm{mm}^{2}\right)$ [9-12] and led to the destruction of the heat exchanger tubes.

Insufficient control of the finished after casting air fan part after destruction was investigated in the work to assess the cause of the destruction of the impeller blade, which is one of the components of the inlet guide vane for controlling the compressor power of the refrigeration system [13]. During the operation the impeller blade was destroyed (Fig. 1).

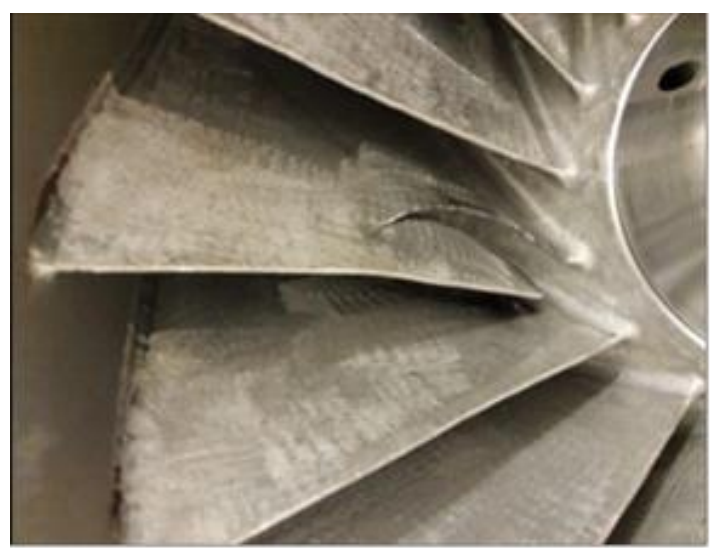

Fig. 1: External view of impeller blade destruction.

The impeller operates at a temperature not higher than $35^{\circ} \mathrm{C}$ and a rotation speed of up to $9500 \mathrm{rpm}$.

In the area where the blades are connected to the shaft attachment sleeve, each blade experiences tensile and bending loads. The impeller is made of silumin.

The research of fractography, relief and roughness was carried out in a similar way [8].

In Fig. 2 shows a photograph of impeller blade destruction surface in the secondary electron mode (the contrast is determined by the topography). It can be seen that the crack surface has a dark color and a slate macro-relief, reflecting alloy structure. The morphology of service fracture surface from the source zone to the zone of static fracture formed during crack opening is the same and is represented by a brittle transcrystalline cleavage oriented along the crystallographic planes of the $\alpha$-phase.

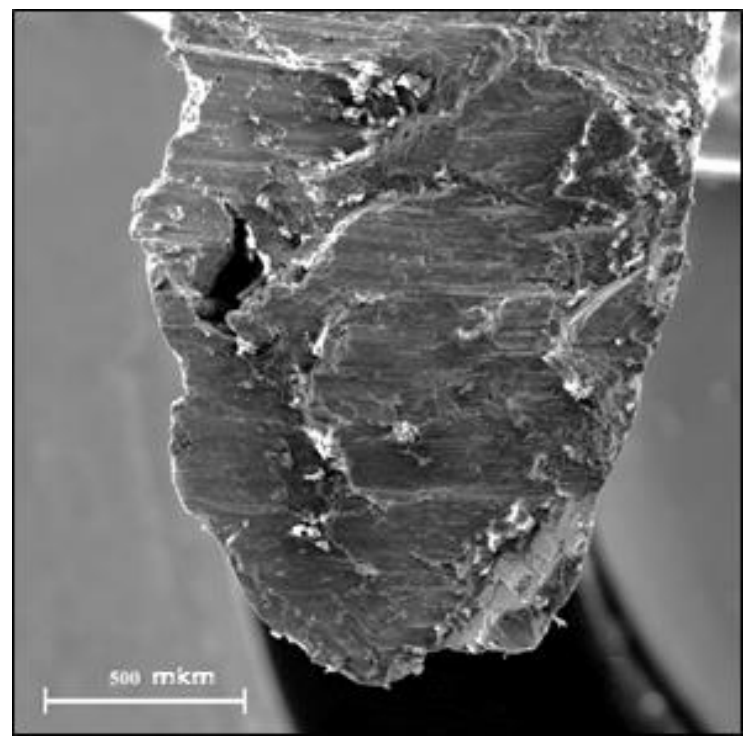

(A)
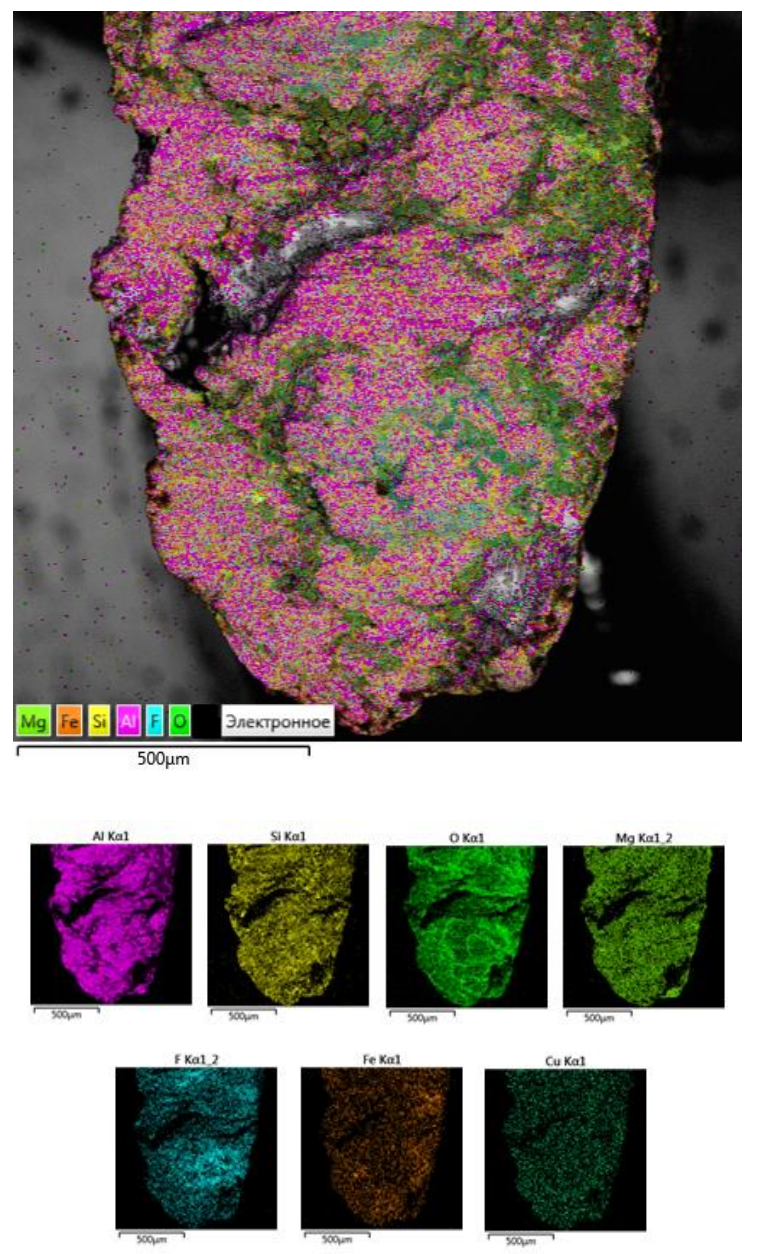

(B) 
Fig. 2: Fracture surface of the impeller blade (A), Spectral mapping of the fracture surface of the impeller blade (B).

The focal zone is located on the edge of the blade and contains metallurgical defects in the form of a void (Fig. 2). Voids are a typical type of rejects in the place of pouring the melt by centrifugal casting. As for the inclusions, it can be concluded from the results of X-ray spectral microanalysis that these are aluminum oxides and residues of aluminum fluorides.

The presence of aluminum fluorides in the focal zone of destruction is due to the fact that in the production of aluminum alloys, a fluoride-chloride electrolyte is often used as an electrolyte in the electrolytic refining of aluminum [14].

As a result of the impeller blade airfoil surface roughness study, traces of machining were found near the crack. Studies have shown that the surface roughness of the material far from the crack is $\mathrm{Ra}$ $40 \mu \mathrm{m}$. At the same time, the roughness near the crack is $\mathrm{Ra}-129 \mu \mathrm{m}$, that is, 3 times more.

The destruction of the impeller blade during operation occurred due to the initiation and propagation of a crack under the influence of an external load. The cause of crack initiation is the large heterogeneity of the cast material. The crack originated near the pore (the void formed during the casting of the product) and the accumulation of aluminum oxides and fluorides. This heterogeneity is due to the peculiarities of metallurgical production $[15,16]$.

To exclude voids and foreign inclusions, each casting must be subjected to non-destructive testing (X-ray, ultrasonic or current-vortex methods).

When operating the installations for the preparation and processing of oil, the compositions of the media significantly deviate from the regulatory ones. As an example of this kind of violations, the premature failure of the pipes of the furnace coils and the occurrence of an emergency and shutdown of the furnace unit of the pyrolysis unit of hydrocarbon raw materials in the ethylene production are considered [17].

The nature of the metal destruction of pyrolysis furnaces pipes made of passivated heat-resistant steel 06Kh16N15M2G2TFR is intergranular corrosion cracks, which can be caused by alkaline corrosion cracking (CC) of austenitic steels. In alkalis, $\mathrm{CC}$ is caused by the passivation of the main surface of the metal and the destruction of passive protective films along the grain boundaries on the carbon-containing phase of the alloy, where impurities are most present. Alkaline CC develops most intensively at a level of tensile stresses close to the yield point [18].
The sensitivity of steel to sodium hydroxide is based on the amphoteric nature of iron oxides, i.e. iron oxides dissolve at both low and high $\mathrm{pH}$ values. Substances with a high $\mathrm{pH}$ value, in particular sodium hydroxide at high temperatures, dissolves magnetite:

$$
4 \mathrm{NaOH}+\mathrm{Fe}_{3} \mathrm{O}_{4} \rightarrow 2 \mathrm{NaFeO}_{2}+\mathrm{Na}_{2} \mathrm{FeO}_{2}+2 \mathrm{H}_{2} \mathrm{O}
$$

After dissolving the protective film of magnetite, sodium hydroxide can react directly with iron atoms [19].

$$
\mathrm{Fe}+2 \mathrm{NaOH} \rightarrow \mathrm{Na}_{2} \mathrm{FeO}_{2}+\mathrm{H}_{2}
$$

To establish destruction causes, studies were carried out on a $06 \mathrm{Kh} 16 \mathrm{~N} 15 \mathrm{M} 2 \mathrm{G} 2 \mathrm{TFR}$ steel sample cut from the tube of the radiant section of the pyrolysis furnace.

The chemical composition was determined on a JEOL JSM 840 scanning electron microscope equipped with an INCA Energy 350 energydispersive (EDS) spectrometer. X-ray structural analysis of the samples was carried out on a DRON$3 \mathrm{M} X$-ray diffractometer. The microstructure was investigated on an Axiotech optical microscope (Karl Zeiss). The microstructure parameters were determined using the Kslite program.

$\mathrm{X}$-ray diffractometer determined the composition of deposits on the inner surface of a pipe sample, which is based on carbides, metal oxides penetrating to a depth of 100 microns (Fig. 3A), which indicates a significant destruction of the surface due to corrosion and the formation of coke deposits. A decrease in the amount of austenitic phase on the inner surface as compared to the outer one is shown. The pipe is heated on the outer surface, that is, it is most exposed to gas corrosion, but the development of local corrosion and cracks occurs on the inner surface of the pipe, that is, the environment inside the chimney affects the steel structure and intense corrosion.

Prepared thin section of the sample of the end surface of the research pipe with a step of $10 \mu \mathrm{m}$ from the inner surface of the pipe to the outer one. The results of experiments to determine the sodium content in the near-surface zone of the metal are shown in the graph (Fig. 3B). 


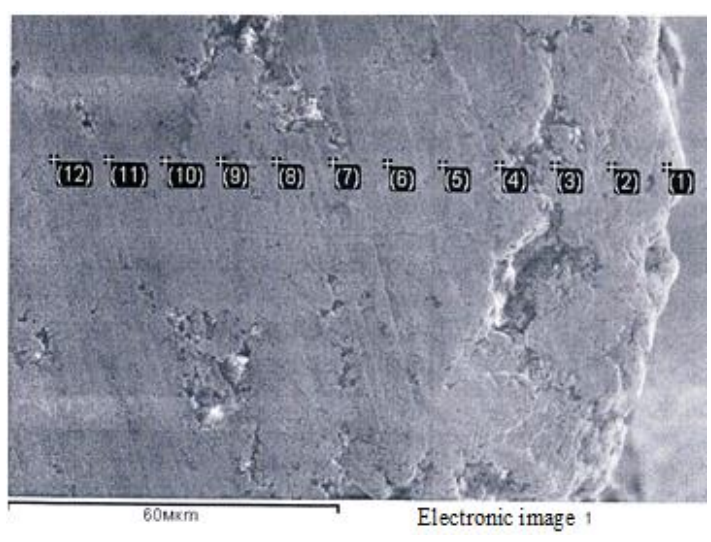

(A)

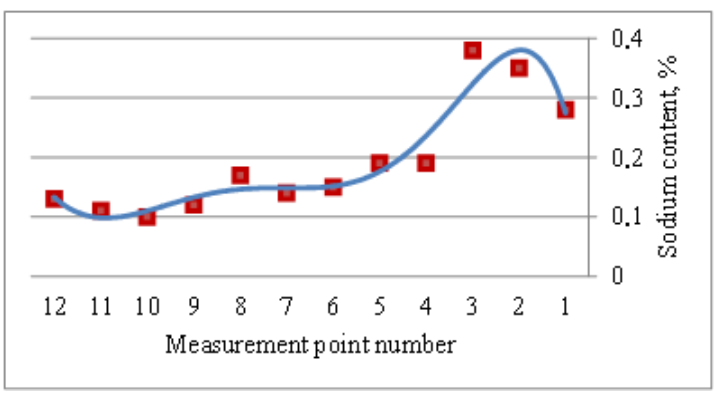

(B)

Fig. 3: Change in sodium concentration (A) along the profile (B) of the chimney section.

The results of determining the sodium content in the near-surface zone of the metal are shown in the graph (Fig. 3B).

Fig. 3 clearly shows that the surface in contact with the vapor-hydrocarbon medium containing alkali $(\mathrm{NaOH})$ has a high sodium concentration. Considering that the surface has been repeatedly washed with water, and sodium is well hydrated, there is little of it on the surface itself, and in the metal itself sodium is presented in the form of a bound and chemically stable compound. Thus, the heat-resistant steel collapsed due to the high sodium concentration in the heated environment. Reducing the amount of supplied alkali will significantly increase the overhaul period of the furnace operation.

The use of gas turbojet engines (GTE) in the transport of hydrocarbons is becoming more and more widespread. During their operation, the phase composition of the alloys and the chemical composition of the surface layers of the parts change. Determining the terms of safe operation of expensive high-loaded products, such as gas turbines, is an important material science problem [20].

Determination of the causes of damage to parts of a high-pressure compressor (HPC) makes it possible to ensure resource reliability, as well as to correct the phase structure of newly created alloys [21], to improve the technologies for the production of critical GTE parts [22]. In the works on the study of titanium alloys [23] the possibility of titanium corrosion is not taken into account. Determination of the destruction mechanism was carried out for damage to the HPC disk of a gas turbine unit used for pumping natural gas. The disc is made of VT8 titanium alloy. Service life of a part in the engine before the start of destruction $<30,000 \mathrm{~h} /$.

The determination of the metal composition was carried out on an Agilent 5100 ICP-OES, LECO CS600, LECO TS-600, RHEN 602 spectrometer; fractography and microstructure were investigated using a JSM-6490LV microscope. The study of the phase structure was carried out on a Tecnai G2 F20 S-TWIN TMP.

Fig. 4 shows the locations of fatigue cracks localization on the disk, which led to significant costs for replacing it, determining the causes of failure and, as a result, changing the manufacturing technology.

The outer diameter of the disc is coated with silver, which in some places is almost completely erased and in these places on the surface of the chamfer there are dark spots and cracks (Fig. 4).

Evaluation of the microstructure and composition of the disc metal confirmed compliance with the alloy requirements. No structural defects and microinclusions were found in the sample.

The study of the fractographic features of the alloys was carried out on samples cut along the cracks (Fig. 4). It was found that corrosion destruction and the formation of both cracks began at the outer edge of the disk. The inner cavity of the crack is covered with oxides. The morphology of the fracture in the area where the corrosive environment was exposed is represented by the facet microrelief, which indicates the brittle nature of the fracture. A static dolly testifies to the viscous nature of destruction.

EDX found that the chemical composition on the surface of the disk sample, as well as on the surface of the cracks, contains silver, VT8 elements, as well as oxygen and chlorine.

In order to determine the mechanisms of disk destruction, a cyclic loading test was carried out: a fatigue crack was grown on the sample, into which, under a static load, a 3\% sodium chloride solution was supplied for 2 hours, then the sample was kept in a desiccator to remove moisture and tested on a pendulum impact machine. 


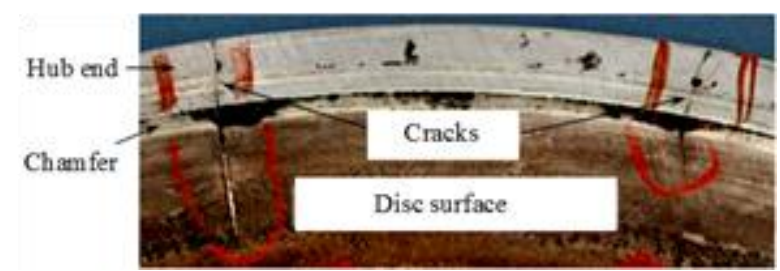

Fig. 4: Cracked disc rims

The fracture photograph shows the following zones (Fig. 5 a): fatigue crack propagation in air; crack development zone in the medium $3 \%$ sodium chloride solution; static downhole in the air. In this case, the first zone has characteristic fatigue grooves (Fig. 5 b). Failure in the second zone is represented by a brittle microrelief (Fig. 5c), similar to the operational one. The third zone has a structure similar to the first (Fig. 5 d).

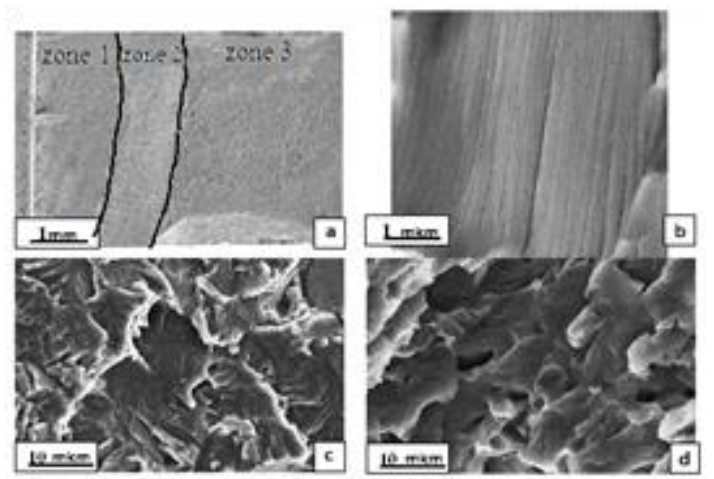

Fig. 5: View of the fracture and fractography of the VT8 sample: a - three zones on the fracture surface of the VT8 sample; b - zone 1; c - zone 2; $\mathrm{d}$ - zone 3.

Tests of VT8 alloy specimens for resistance to the rate of crack growth in a corrosive environment showed the alloy's tendency to embrittlement and stress corrosion cracking in chloride-containing media, with the formation of a microrelief (Figs. $5 \mathrm{c}$, d), corresponding to a brittle one that is not typical for fracture in air, but identical to operational fracture (Fig. 4). Thus, the destruction occurred due to the embrittlement of the metal in the process of electrochemical corrosion in molten nickel chlorides, intensified by galvanic corrosion in the presence of a silver coating film.

\section{Conclusion}

In the process of creating equipment, the experience of operating materials in conditions of aggressive action of corrosive environments must be taken into account and the reasons leading to the destruction of materials must be thoroughly investigated. The authors have developed a sequence for determining the causes of the destruction of various parts:

1) Assessment of operating conditions;
2) Determination of the nature of the part destruction by methods of X-ray diffraction, $\mathrm{X}$ ray phase analysis and electron emission microscopy;

3) Determination of corrosion products composition, diffusion of elements of the corrosive medium into the depth of the metal and alloying elements in the volume of the metal;

4) The phase inversion in the alloy is investigated to analyze possible technological overheating or excessive mechanical loads of the part.

5) Based on the analysis of the data obtained and, if necessary, additional experiments on modeling the processes during the operation of the part, the reasons for the destruction of the part are determined and recommendations are given to prevent such destruction in the future.

Preliminary testing of materials with simultaneous exposure to a variable load, a corrosive environment and contact of materials in the mated parts is necessary in order to ensure the equipment is going to work in the long run and to prevent breakdowns and accidents.

\section{Appendix}

Appendixes, if needed, appear before the acknowledgment.

\section{Acknowledgment:}

«The work was performed using the equipment of the center shared research facilities "Climate Tests" of FSUE «VIAM».

\section{References:}

[1] J.-W. Yeh, S.-K. Chen, S.-J. Lin, J.-Y. Gan, T.S. Chin, T.-T. Shun, C.-H. Tsau and S.-Y. Chang, Nanostructured High-Entropy Alloys with Multiple Principal Elements: Novel Alloy Design Concepts and Outcomes, Advanced Engineering Materials, Vol.6, No.5, 2004, pp. 299-303. DOI: 10.1002/adem.200300567.

[2] J. Sieniawski, W. Ziaja, K. Kubiak and M. Motyka, Microstructure and Mechanical Properties of High Strength Two-Phase Titanium Alloys. Titanium alloys - Advances in Properties Control. Microstructure and mechanical properties of high strength twophase titanium alloys, In Tech, 2013. DOI: $10.5772 / 56197$.

[3] Mothana Ghazi Kadhim and Mushtaq Albdiry, A Critical Review on Corrosion and its Prevention in the Oilfield Equipment, Journal 
of petroleum research and studies, Art. 14, 2017. DOI: 10.52716/jprs.v7i2.195.

[4] S. Kanaun and A. Markov, Discrete and ThreeParameter Models of Hydraulic Fracture Crack Growth, WSEAS Transactions on Applied and Theoretical Mechanics, No.12, 2017, pp.147156.

[5] I. Enescu, Some Researches Regarding Stress Intensity Factors in Crack Closure Problems, No.13, 2018, pp. 187-192.

[6] R. Jarkovsky, P. Cyrus and S. Major, Model of Fatigue Life Degradation of Crankshaft, WSEAS Transactions on Applied and Theoretical Mechanics, No.13, 2018, pp. 175180.

[7] , A. Arutyunyan, R. Arutyunyan and R. Saitova, The Criterion of High-Temperature Creep of Metals Based on Relative Changes of Density, WSEAS Transactions on Applied and Theoretical Mechanics, No.14, 2019, pp. 140144.

[8] R. Zh. Akhiyarov, A. B. Laptev, D. A. Movenko and N. A. Belova, Investigation of Abnormally Low Corrosion Resistance of Pipe Steel of Heat Exchange Equipment for Oil Refining, Oil industry, No. 1, 2016, pp. 118121.

[9] I. I. Reformatskaya, I. G. Rodionova, Yu. A. Beilin, et al, The Role of non-Metallic Inclusions and Microstructure in the Process of Local Corrosion of Carbon and Low-Alloy Steels, Protection of Metals, Vol.40, No.5, 2004, pp. 498-503.

[10] A. I. Zaitsev, B. M. Mogutnov and E. Kh. Shakhpazov, Physical Chemistry of Metallurgical Slags, Interkontakt Nauka, 2008.

[11] G. I. Kotelnikov, D. A. Movenko, A. V. Pavlov and S. A. Motrenko, Model of the Distribution of Tensile and Compressive Stresses in Metal Around Calcium-Containing Non-Metallic Inclusions in Aqueous Media, Izvestiya Vuzov, Ferrous Metallurgy, No.3, 2014, pp. 10-16.

[12] L. V. Morozova and I. V. Iskhodzhanova, Investigation of the Regularities of Changes in the Surface Relief of Aluminum-Lithium Alloys by the Method of Laser Microscopy, ARRIAM Works: Electron. Scientific and Technical Magazine, Vol.10, No.8, 2014. Available: http://www.viam-works.ru (date of access: 11.04.2019). doi 10.18577 / 2307-60462014-0-10-8-8.

[13] I. P. Zhegina, V. B. Grigorenko, L. V. Morozova and M. A. Fomina, Investigation of Changes in the State of Surface Layers of Aluminum-Lithium Alloys 1441 and v-1469 Under the Complex Action of Stresses and a Corrosive Environment, Aviation Materials and
Technologies, No.S4, 2014, pp. 65-69. DOI 10.18577 / 2071-9140-2014-0-s4-65-69.

[14] A. V. Grinevich, Yu. S. Rumyantsev, L. V. Morozova and A. L. Terekhin, Investigation of the Fatigue Life of Aluminum Alloys 1163-t and V95o.ch. -T2 After Surface Hardening Aviation Materials and Technologies, No.S4, 2014, pp. 93-102. DOI 10.18577 / 2071-91402014-0-s4-93-102.

[15] E. N. Kablov, Innovative Developments of FSUE "VIAM" SSC RF for the Implementation of "Strategic Directions for the Development of Materials and Technologies for their Processing for the Period Up to 2030", Aviation Materials and Technologies, Vol.1, No.34, 2015, pp. 333. DOI: 10.18577 / 2071-9140-2015-0-1-3-33.

[16] E. N. Kablov, O. V. Startsev and I. M. Medvedev, Review of Foreign Experience in Corrosion Research and Corrosion Protection Products, Aviation Materials and Technologies, No.2, 2015, pp. 76-87. DOI: 10.18577 / 20719140-2015-0-2-76-87.

[17] E. N. Kablov, N. A. Nochovnaya, P. V. Panin, E. B. Alekseev and A.V. Novak, Investigation of the Structure and Properties of HeatResistant Alloys Based on Titanium Aluminides With Microadditions of Gadolinium, Materials Science, No.3, 2017, pp. 3-10.

[18] A. B. Laptev, D. A. Movenko and R. Zh. Akhiyarov, Investigation of the Cause of the Operational Destruction of the Impeller Blade of the Compressor of the Refrigeration Supply System, Practice of Anti-Corrosion Protection, Vol.1, No.83, 2017, pp. 32-38.

[19] A. B. Laptev, E. V. Nikolaev and M. R. Pavlov, Investigation of the Causes of Destruction of Steel 06Kh16N15M2G2TFR of the Furnace Coil and the Development of a Protection Method, Practice of Anti-Corrosion Protection, Vol.1, No. 83, 2017, pp. 39-44.

[20] T. V. Pavlova, O. S. Kashapov, A. R. Kondratyeva and V. S. Kalashnikov, Possibilities for Expanding the Scope of VT8-1 Alloy for Compressor Disks and Impellers, Proceedings of VIAM: Electron, Scientific and Technical Magazine, No.3, Art.05, 2016. Available: http://www.viam-works.ru (date accessed: 15.10.2018). DOI 2307-6046-2016-03-5-5.

[21] M. R. Orlov, Yu. A. Puchkov, S. A. Naprienko and A. V. Lavrov, Investigation of Operational Destruction of a Fan Blade of an Aircraft Gas Turbine Engine Made of VT3-1 Titanium Alloy, Titan, Vol.4, No.46, 2014, pp. 23-30.

[22] S. P. Lynch, Hydrogen Embrittlement (HE) Phenomena and Mechanisms, ed. V. S. Raja, T. 
Shoji et al., Stress Corrosion Cracking, Cambridge: Woodhead Publishing, 2011.

[23] Š. Major, M. Ržička and P. Cyrus, Fatigue Life Prediction of Titanium Implants with Titanium Dioxide Surface, WSEAS Transactions on Applied and Theoretical Mechanics, Vol.13, No.2, 2018, pp. 8-15.

\section{Creative Commons Attribution}

\section{License 4.0 (Attribution 4.0}

\section{International, CC BY 4.0)}

This article is published under the terms of the Creative Commons Attribution License 4.0

https://creativecommons.org/licenses/by/4.0/deed.en US 